\title{
Centerline Extraction for Image Segmentation Using Gradient and Direction Vector Flow Active Contours
}

\author{
Shuqun Zhang ${ }^{1}$, Jianyang Zhou ${ }^{2}$ \\ ${ }^{1}$ Department of Computer Science, College of Staten Island, City University of New York, New York, USA; ${ }^{2}$ Department of Elec- \\ tronic Engineering, Xiamen University, Xiamen, China. \\ Email: shuqun.zhang@csi.cuny.edu, zhoujy@xmu.edu.cn
}

Received September $19^{\text {th }}, 2013$; revised October $19^{\text {th }}$, 2013; accepted October $26^{\text {th }}, 2013$

Copyright (C) 2013 Shuqun Zhang, Jianyang Zhou. This is an open access article distributed under the Creative Commons Attribution License, which permits unrestricted use, distribution, and reproduction in any medium, provided the original work is properly cited.

\begin{abstract}
In this paper, we propose a fast centerline extraction method to be used for gradient and direction vector flow of active contours. The gradient and direction vector flow is a recently reported active contour model capable of significantly improving the image segmentation performance especially for complex object shape, by seamlessly integrating gradient vector flow and prior directional information. Since the prior directional information is provided by manual line drawing, it can be inconvenient for inexperienced users who might have difficulty in finding the best place to draw the directional lines to achieve the best segmentation performance. This paper describes a method to overcome this problem by automatically extracting centerlines to guide the users for providing the right directional information. Experimental results on synthetic and real images demonstrate the feasibility of the proposed method.
\end{abstract}

Keywords: Image Segmentation; Active Contours; Gradient Vector Flow; Direction Vector Flow

\section{Introduction}

Image segmentation is to separate an object of interest from the rest of an image. It is one of the most important and yet difficult problems in image processing and computer vision, which has been addressed by many algorithms, among which, active contours, also known as snakes, have been extensively studied and used since they were first introduced by Kass et al. [1]. Active contours are dynamic curves modeled to evolve towards the object boundary by minimizing some given functional energy. Since designing external force is the key for the performance of active contours, many external forces have been proposed [2-7] such as Cohen and Cohen's balloon force [2], Park and Chung's virtual electric field [3], Xie and Mirmehdi's magnetostatic field [4], Sum and Cheung's boundary vector field [5], $\mathrm{Li}$ and Acton's vector field convolution [6], and $\mathrm{Xu}$ and Prince's gradient vector flow (GVF) field [7]. Among them, the GVF is the most widely investigated external force field, which is computed as a diffusion of the gradient vectors of image edge map. The GVF field has the advantages of larger capture range and the ability of pushing the fronts of active contours into concave boundaries over the traditional exter- nal force. Due to these advantages and being easily extended, many works have followed the original GVF model to further improve the performance. For example, the generalized GVF [8] improved the convergence of active contours to long, thin boundary indentations [8]. Xie et al. [9] modified the GVF, so it can be more robust to image noise. Ray et al. [10] incorporated the motion direction into the GVF model for tracking rolling leukocytes. The GVF field has been also modified by considering the directional information of image edge to make active contours discern the image edge with different directions [11].

Prior information is important for image segmentation algorithm development if it is available and can be integrated into the algorithm. In the past, various priors such as object shape, size, position, and motion direction have been incorporated to improve the performance of active contour models. However, since these priors are usually not easily obtained or estimated for all images, they are useful only for some particular types of images or applications. Recently, a general method for providing prior directional information was proposed [12], which is implemented simply by drawing a few directional lines towards the desired evolving direction after the normal 
contour initialization step. Correspondingly, a novel external force called gradient \& direction vector flow (G \& DVF) [12] was developed to seamlessly integrate the GVF field and the direction vector field produced by the directional lines drawn by a user. This is a very simple method for providing prior information and can be used for any images. It has been also demonstrated that the $\mathrm{G}$ \& DVF field can significantly improve the performance of the GVF model on segmenting images with complex object shape, and can alleviate the requirement that the initial contour must be very close to the true object boundary in order to obtain a good performance. However, since the directional information is provided by user mouse clicks, it is inconvenient particularly for those inexperienced users who might not be able to find the best place to draw the directional lines on the image for the algorithm to be effective. This paper proposes a method to automatically extract centerlines of objects and concaves, which can be used to guide the users where the direction lines should be drawn on the image to obtain the best segmentation performance. The feasibility of the proposed method is demonstrated by experiments tested on both synthetic and real images.

The rest of this paper is organized as follows. Section 2 reviews the related active contour models and describes the proposed centerline extraction method. Then, the experimental results and discussion are provided in Section 3. Finally, we present our conclusions and future work.

\section{Method}

\subsection{Classical Active Contour}

The classical active contour [1] is usually modeled as a dynamic curve $\mathbf{x}(s)=[x(s), y(s)], s \in[0,1]$, that evolves from an appropriate initial position to the object boundary by minimizing the following energy functional

$$
E_{\mathrm{AC}}(\mathbf{x})=\frac{1}{2} \int_{0}^{1} \alpha\left|\mathbf{x}^{\prime}(s)\right|^{2}+\beta\left|\mathbf{x}^{\prime \prime}(s)\right|^{2} \mathrm{~d} s+\int_{0}^{1} E_{\mathrm{Ext}}(\mathbf{x}(s)) \mathrm{d} s
$$

where $\alpha$ and $\beta$ are positive weighting parameters. The first and second terms are the internal energy and external energy, which are used to regularize the smoothness of the curve $\mathbf{x}$ and attract the curve $\mathbf{x}$ toward the object boundary, respectively. The function $E_{\mathrm{Ext}}$ in the external energy is usually defined as the negative intensity of the image edge map $f$, which is computed by first smoothing the image $I$ with a Gaussian kernel followed by a gradient to enhance the boundaries as

$$
E_{\text {Ext }}(x, y)=-f(x, y)=-\left|\nabla\left[G_{\sigma}(x, y) * I(x, y)\right]\right|
$$

where $G_{\sigma}(x, y)$ denotes a Gaussian filter with standard deviation $\sigma$. The minimization of $E_{\mathrm{AC}}$ can be achieved by evolving the front of the active contour dynamically as a function of parameter $s$ and artificial time $t$ given by

$$
\mathbf{x}_{t}(s, t)=\left[\alpha \mathbf{x}^{\prime \prime}(s, t)-\beta \mathbf{x}^{\prime \prime \prime \prime}(s, t)\right]-\nabla E_{\mathrm{Ext}},
$$

where the first term and the second term are generally called the internal force and the external force, respectively. The classical active contour has poor performance in image segmentation, and thus many improvements have been proposed, among which the GVF has received many attentions.

\subsection{Gradient Vector Flow}

The GVF is an external force proposed to overcome the two main drawbacks of the traditional active contours: limited capture range and poor convergence to concave boundary. It substitutes the traditional external force $-\nabla E_{\text {Ext }}$ in Equation (3) with the GVF field $\mathbf{v}(x, y)=[u(x$, $y), v(x, y)]$, which is derived from the diffusion of the gradient vectors of the image edge map, and is set to minimize the following energy functional

$$
E_{\mathrm{GVF}}(\mathbf{v})=\iint \mu|\nabla \mathbf{v}|^{2}+|\nabla f|^{2}|\mathbf{v}-\nabla f|^{2} \mathrm{~d} x \mathrm{~d} y .
$$

In Equation (4), the first term in the integrand is used to smooth the vector field $\mathbf{v}$, and has the effects of making the force field robust to image noises and enlarging the capture range of the force field, while the second term is the data fidelity term that keeps $\mathbf{v}$ being equal to the gradient vector of the edge map $\nabla f$ where the magnitude of $\nabla f$ is relatively large. $\mu$ is the smoothness regularization parameter.

The GVF field can be obtained by solving the following Euler-Lagrange equation

$$
\mathbf{v}_{t}=\mu \nabla^{2} \mathbf{v}(t)-(\mathbf{v}-\nabla f)|\nabla f|^{2},
$$

which is derived from the variational minimization of the energy functional $E_{\mathrm{GVF}}$ with respect to $\mathbf{v}$.

\subsection{Gradient and Direction Vector Flow}

Although the GVF has some improvement over the classical active contour, it still has poor performance in segmenting images with complex object shapes. Prior information such as object shape, location and size are very useful in further improving the image segmentation performance, but they are usually limited to some particular images or applications. A much more general, simple and effective way for providing prior is to give the correct evolving direction to active contours. Based on this observation, we recently proposed a new external force called G\&DVF [12] to further help the GVF active contour converge to the correct object boundary by integrating the GVF and prior directional information, in which the directional information is obtained by drawing a few 
directional lines to point to the direction that the front of active contour should evolve. The proposed G \& DVF minimizes the following energy functional

$$
E_{\mathrm{G} \& \mathrm{DVF}}(\mathbf{v})=E_{\mathrm{GVF}}(\mathbf{v})+\eta E_{\mathrm{DVF}}(\mathbf{v}),
$$

where $E_{\mathrm{GVF}}$ and $E_{\mathrm{DVF}}$ denote the GVF functional and the direction vector flow (DVF) functional that is generated by the directional lines, respectively. The functional $E_{\mathrm{DVF}}$ is defined as

$$
E_{\mathrm{DVF}}(\mathbf{v})=\iint|\mathbf{w}|^{2}|\mathbf{v}-\mathbf{w}|^{2} \mathrm{~d} x \mathrm{~d} y,
$$

where $\mathbf{w}$ is the DVF filed obtained by drawing a direction line in the image, and represented as

$$
\mathbf{w}(x, y)= \begin{cases}\frac{\mathbf{b}-\mathbf{a}}{|\mathbf{b}-\mathbf{a}|}, & \text { if }(x, y) \text { is on } \overrightarrow{\mathbf{a b}} \\ \mathbf{0}, & \text { otherwise, }\end{cases}
$$

where $\mathbf{a}$ and $\mathbf{b}$ denote the starting and the end point of the directional $\overrightarrow{\mathbf{a b}}$ line, respectively. If multiple lines are needed, we can simply add the corresponding direction vector fields together.

The DVF functional in Equation (7) is designed to keep $\mathbf{v}$ being equal to $\mathbf{w}$ where the norm of $\mathbf{w}$ is relatively large. The influence of the directional lines on the external force field $\mathbf{v}$ is controlled by the parameter $\eta$. The $\mathrm{G}$ \& DVF field can be obtained by solving the following Euler-Lagrange equation

$$
\mu \nabla^{2} \mathbf{v}-(\mathbf{v}-\nabla f)|\nabla f|^{2}-\eta(\mathbf{v}-\mathbf{w})|\mathbf{w}|^{2}=0 .
$$

The gradient decent of Equation (9) can be obtained by

$$
\mathbf{v}_{t}=\mu \nabla^{2} \mathbf{v}-(\mathbf{v}-\nabla f)|\nabla f|^{2}-\eta(\mathbf{v}-\mathbf{w})|\mathbf{w}|^{2},
$$

with parameterizing the descent direction by an artificial time $t>0$. The solution of Equation (10) can be obtained when the iterative process of the gradient decent is at the steady state.

Figure 1 shows an example of the usage and effectiveness of the G \& DVF. The object to be segmented is a synthetic swirl shape image as shown in Figure 1(a). Figures 1(b) and (c) give the segmentation results of the GVF and G \& DVF, respectively. It is seen from Figure 1(b) that the GVF active contour cannot conform to the object boundary from the circle-shaped initialization outside of the object. The contour is stuck at the entry of the object. The rolling concave in the object is too difficult for the GVF active contour to handle. However, if we simply place three short lines inside the rolling concave pointing inward as shown in Figure 1(a), the G \& DVF can easily move the initial contour into the rolling concave, as shown in Figure 1(c).

\subsection{Centerline Extraction for G \& DVF}

The G \& DVF has been proven to provide much better

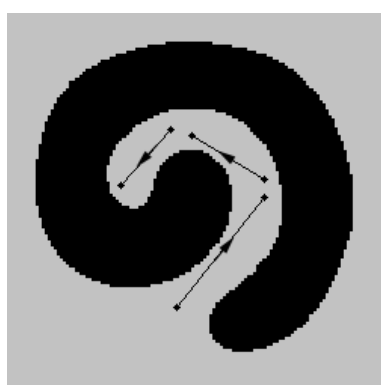

(a)

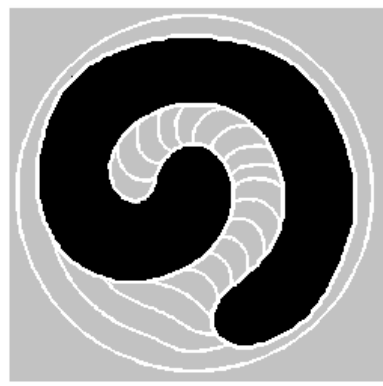

(c)

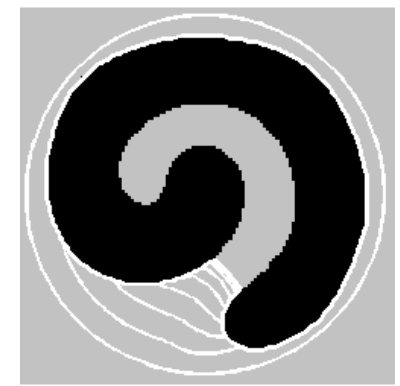

(b)

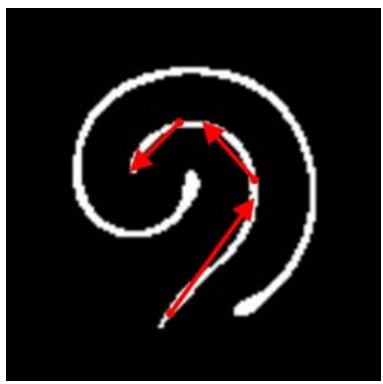

(d)
Figure 1. Demonstration of the usage and effectiveness of the G \& DVF: (a) The image to be segmented; (b) Front propagation of the original GVF active contour; (c) Front propagation of the G \& DVF active contour; (d) The extracted centerlines and the produced directional lines.

performance than the GVF and other active contour models, especially when dealing with complex object shapes and weak-edge-leakage [12]. However, it is inconvenient in the sense that the DVF field is produced by user drawing lines. An inexperienced user may have difficulty in finding the best place to draw the lines to achieve the best performance. Since the G \& DVF will have the best segmentation performance if the lines are drawn along the centerline of the object or concave, it would be convenient if the centerline is provided to the user. Hence here we propose to extract the centerlines automatically to help users in using G \& DVF active contours, which is based on the method for detecting saddle/stationary points [13] and curve skeletons [14].

It is well known that the GVF field is a slowly varying field that diffuses from the object boundary toward its center. It has the property that it is smooth in most image domain, except at the ridge of an object and the center of a concave, and along the strong image edge. To extract centerlines of an object or concave, we can utilize the magnitude of the gradient of GVF field, i.e., $|\nabla \mathbf{v}(x, y)|$ which is approximated to zero where the GVF field is smooth, and has a relatively large value in other places. Therefore, we can discern the centerlines as well as the edge points from other smooth GVF field by checking the value of $|\nabla \mathbf{v}(x, y)|$. Since we are interested in extracting centerlines only, we need to further remove the edges after separating the smooth area. To achieve this, 
an edge indicator function $g(x, y)=1 / 1+f(x, y)$ can be used to multiply with the magnitude of the gradient of GVF field since the edge function is very small when the pixel at $(x, y)$ is on the strong edge and has a value of 1 in other places. In summary, centerlines can be extracted by first calculating the product of the magnitude of the gradient of GVF field and the edge indicator function,

$$
k(x, y)=g(x, y)|\nabla \mathbf{v}(x, y)|,
$$

then normalizing it to $[0,1]$,

$$
\bar{k}(x, y)=\left(\frac{k(x, y)-\min (k)}{\max (k)-\min (k)}\right)^{q},
$$

and finally comparing with a threshold

$$
s(x, y)=\left\{\begin{array}{l}
1 \text { if } \bar{k}(x, y)>T, \\
0 \text { else, }
\end{array}\right.
$$

where $T$ is a pre-set threshold between 0 and 1 . In Equation (12), $q$ is the field strength taking a value between 0 and 1 , which can be used to control the centerline strength. Morphological operations such as opening can be applied to process the threshold result

$$
s(x, y) \circ B,
$$

where $B$ is a structuring element, and "o" denotes a morphological opening operation. If necessary, thinning or skeleton algorithm can be further used to make the extracted lines thinner. Once the centerlines are extracted, the G \& DVF users will know where the best place is to draw the directional lines and generate the DVF field. That is, the users can just simply follow the detected centerlines to draw the directional lines. As an example, Figure 1(d) shows the centerline extraction result from Figure 1(a) using the proposed method described above, where lines outside the object support has been removed. The required directional lines (shown in red color) for the G \& DVF can be easily obtained based on Figure 1(d), which are similar to the lines shown in Figure 1(a).

It is noted that the proposed method has very low computational complexity since it simply utilizes the already computed GVF for centerline extraction and there is no much extra calculation needed.

\section{Experimental Results}

To demonstrate the feasibility of the proposed method, we present experiments tested on both synthetic and real images. The image size is $120 \times 120$ pixels for the tested synthetic image, while is $240 \times 240$ pixels for the tested real image. In the experiments, the edge maps for computing the force fields are normalized to the range $[0,1]$. The parameters $\alpha=0.8, \beta=0.0$, and $\mu=0.2$ were chosen for both the G \& DVF and the GVF, and $\eta=1.5$ for the
G \& DVF. The field strength $q=1$ is used. The GVF and G \& DVF active contours use the same circle-shaped initializations in each of our experiments, which are not placed in the neighborhood of the desired object boundary.

Figure 2 shows an experiment tested on a synthetic "S" shape image. The original image is shown in Figure 2(a). The two semi-closed concaves formed in the "S" shape are difficult for the original GVF field to handle and the contour is usually stuck at the entries. This can be seen from Figure 2(b) that shows the segmentation result using the original GVF model. The failure of segmentation using the original GVF is because there are some GVF field vectors pointing outward at the entry of the semi-closed concave as shown in Figure 2(c), which prevents the front of the GVF active contour moving into the semi-closed concave boundary. For better segmenting the "S" shape image, we can use the G \& DVF and draw two directional lines at the entries of the two semi-closed concaves as shown in Figure 2(e) to force the field vector to point inward. The field vector change from outward to inward is demonstrated in Figure 2(d), which shows the G \& DVF field vectors at the entry of the semi-closed concave. The changing of field vector direction helps the front of the G \& DVF active contour exactly propagate into the semi-closed concave as shown Figure 2(f). The two drawn lines shown in Figure 2(e) actually can be extracted using the proposed method. Figure 2(g) shows the detection result of the centerlines using the proposed method. Given Figure 2(g), the G \& DVF users will know where exactly to draw the direction lines, simply following the centerlines as shown in Figure 2(h). Alternatively we can also automatically produce a line by finding the two end points of a detected area.

Another experiment was performed on a real corpuscallosum MRI image as shown on Figure 3(a). The active contour is initialized at one end of the corpus-callosum. We expect that the front of the active contour moves into a hooked concave for exactly conforming to the corpus-callosum boundary. The segmentation result using the original GVF model is given in Figure 3(b), where the GVF field obviously cannot drive the front of the GVF active contour to move into the hooked concave, and the front of the GVF active contour stops moving at the beginning of the hooked concave. With the G \& DVF active contour, simply drawing three directional lines on the image will assist the curve evolution, as shown in Figure 3(c). The corresponding segmentation result by the G \& DVF is shown in Figure 3(d). It successfully drives the front of the active contour to the correct object boundary from the circle-shaped initialization. The obtained different results for these two models can be explained from their vector fields as shown in Figures 3(e) and (f), where the GVF field vectors point to many dif- 


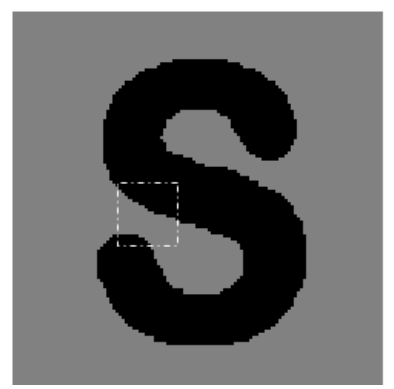

(a)

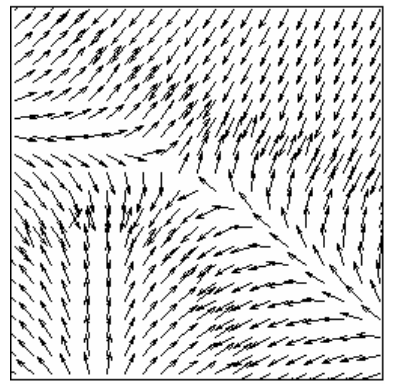

(c)

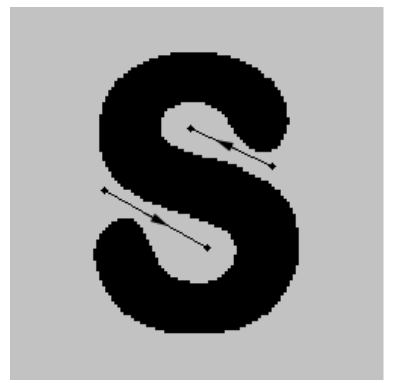

(e)

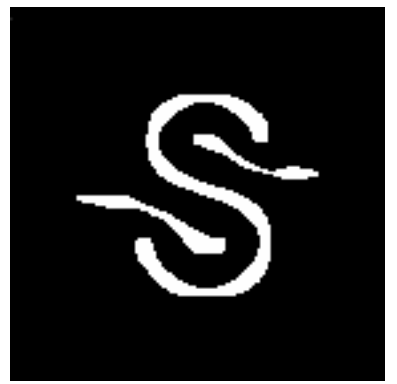

(g)

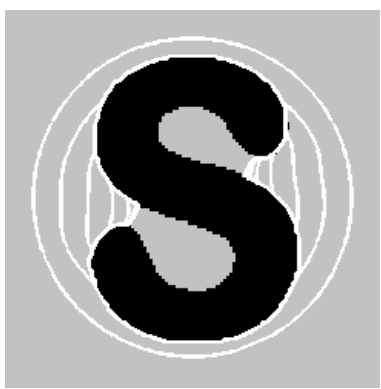

(b)

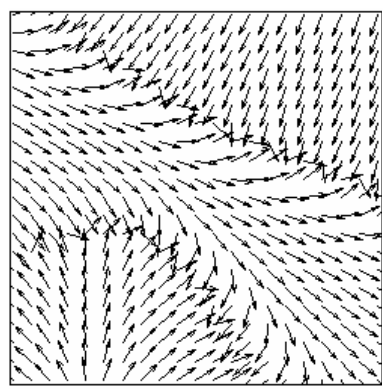

(d)

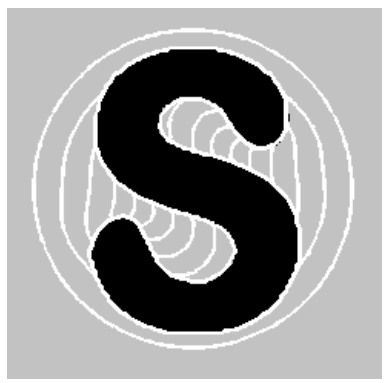

(f)

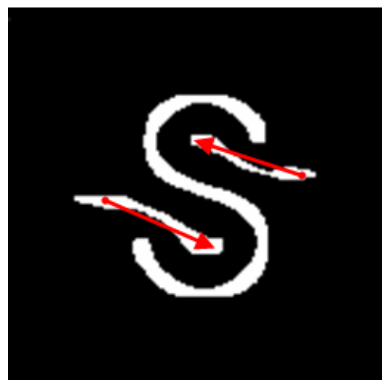

(h)

Figure 2. Experimental results of a synthetic " $S$ " image: (a) The original synthetic image; (b) Front propagation of the original GVF active contour; (c) and (d) The GVF field and the G \& DVF field within the dashed area of (a), respectively; (e) Two directional lines drawn on the image for the G \& DVF model; (f) Front propagation of the G \& DVF active contour; (g) The extracted centerlines; (h) The directional lines produced on the obtained centerlines.

ferent directions while the G \& DVF field vectors point to the same direction because of the force from the three lines. Again we can generate the needed direction lines using the proposed method. The detected centerlines are

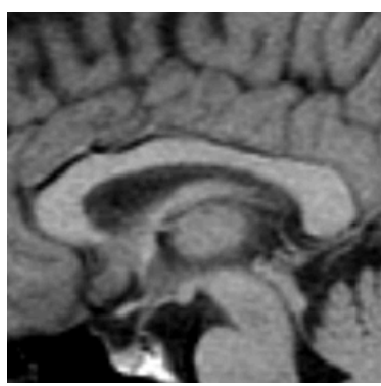

(a)

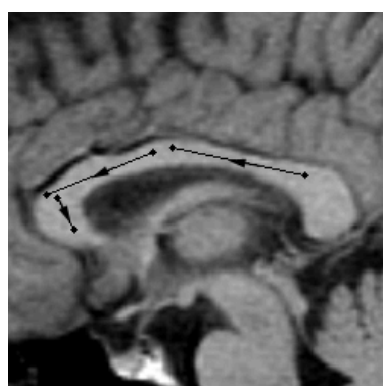

(c)

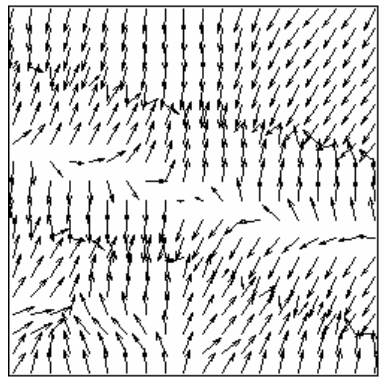

(e)

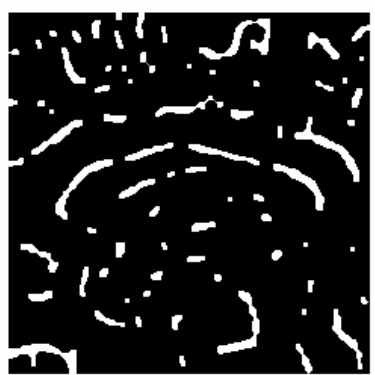

(g)

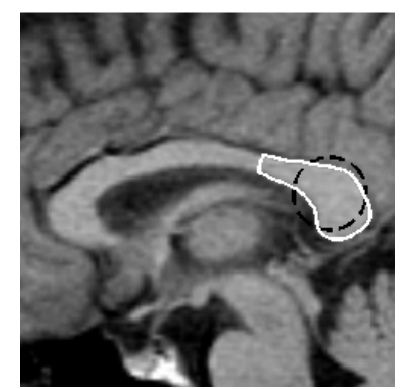

(b)

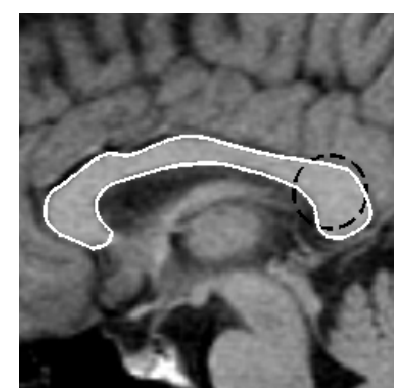

(d)

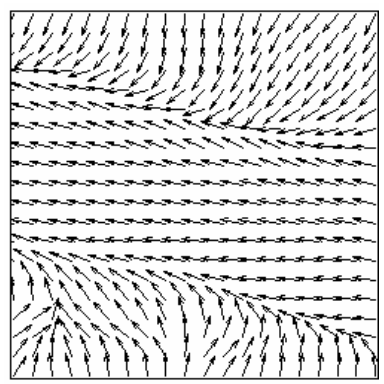

(f)

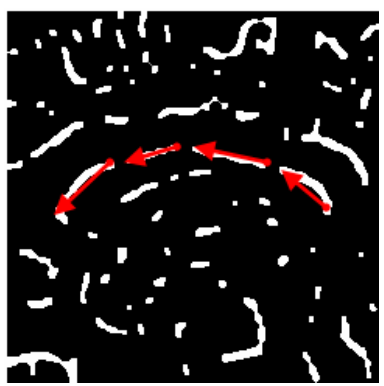

(h)
Figure 3. Experimental results of a corpus-callosum MRI image: (a) The corpus-callosum MRI image; (b) Front propagation of the GVF active contour; (c) Three directional lines drawn on the image for the G \& DVF model; (d) Front propagation of the G \& DVF active contour; (e) The GVF field of a small area in the middle of the corpus-callosum; (f) The G \& DVF field of the same area of the corpus-callosum as in (e); (g) The extracted centerlines; (h) The directional lines produced on the obtained centerlines.

shown in Figure 3(g), and the direction lines can then be obtained from the detected centerlines, as shown in Figure 3(h). 
Figure 4 shows another experiment tested on a real hand image. It is shown in Figure 4(b) that, the GVF active contour cannot conform to the semi-closed concave formed by the fingers, while by simply adding a directional line at the entry of the concave, as shown in Figure 4(a), the G \& DVF active contour can converge to the exact hand boundary. The directional line can be generated based on the centerline of the concave, which is extracted using the proposed method and shown in Figure 4(d).

In the proposed method, the centerline strength can be controlled by changing the value of the field strength $q$ in Equation (12). To study the effect of the field strength on the centerline strength, we vary the value of $q$ and apply Equation (12) on the corpus-callosum MRI image given in Figure 3(a). The results are obtained as shown in Figure 5. It is obviously seen that decrementing the field strength $q$ will enhance the centerline strength.

\section{Conclusion}

The recent reported G\&DVF active contour provides a very good performance in segmenting images with complex object shapes and dealing with the issue of weakedge-leakage. To overcome the problem that the directional information is manually provided in the G \& DVF model, this paper proposes a fast semi-automatic method for producing directional information by extracting cen-

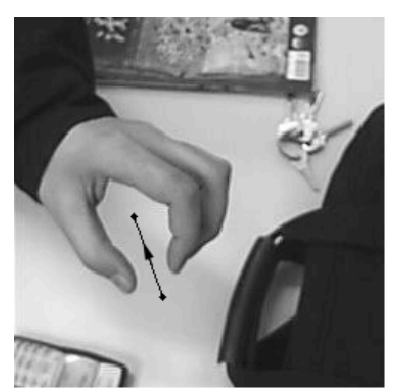

(a)

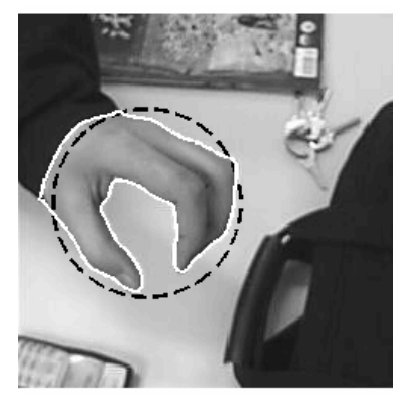

(c)

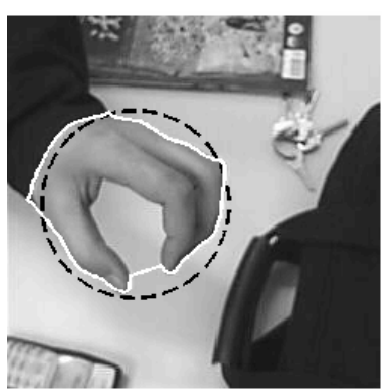

(b)

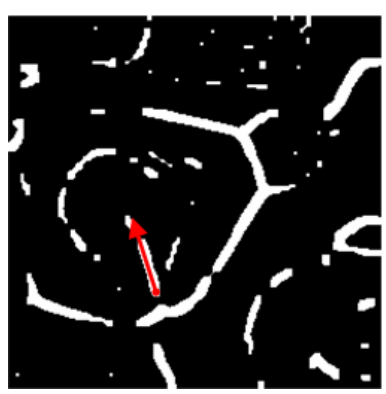

(d)
Figure 4. Experimental results of a hand image: (a) The hand image; (b) Front propagation of the GVF active contour; (c) Front propagation of the G \& DVF active contour; (d) The extracted centerlines and the overlapping directional lines.

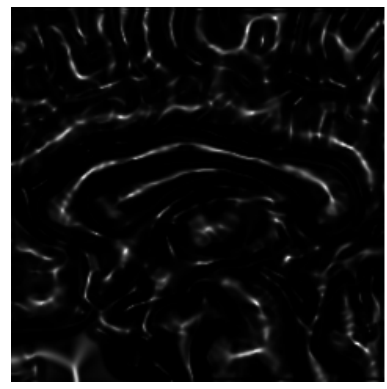

(a)

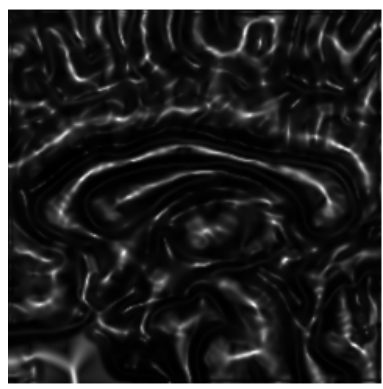

(c)

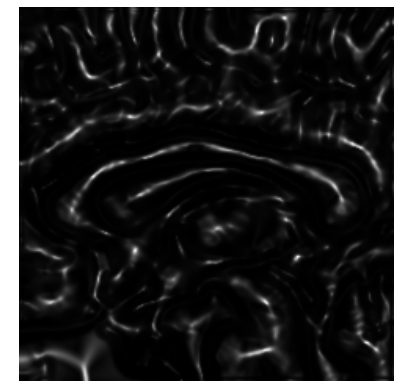

(b)

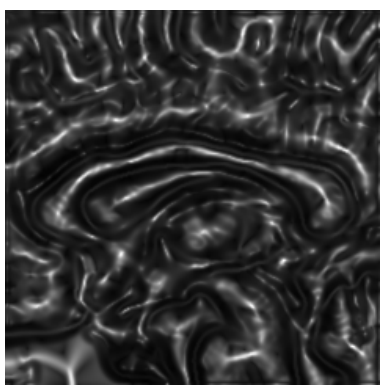

(d)
Figure 5. Effect of the field strength $q$ on the centerline strength: (a) $q=1.0$; (b) $q=0.8$; (c) $q=0.6$; (c) $q=0.4$.

terlines of objects and concaves. The extracted centerlines can effectively guide users to provide the best directional information for the G \& DVF model to achieve the best image segmentation performance. Experimental results show the feasibility of the proposed method. Future work will develop fully automatic method for producing the required directional lines for G \& DVF active contours.

\section{Acknowledgements}

This work was supported by a grant from National Key Technology R\&D Program of MOST of China (2012BAI07B06) and in part by a grant (\# 65394-00-43) from The City University of New York PSC-CUNY Research Award Program.

\section{REFERENCES}

[1] M. Kass, A. Witkin and D. Terzopoulos, "Snakes: Active Contour Models," International Journal of Computer Vision, Vol. 1, No. 4, 1988, pp. 321-331. http://dx.doi.org/10.1007/BF00133570

[2] L. D. Cohen and I. Cohen, "Finite-Element Methods for Active Contour Models and Balloons for 2-D and 3-D Images," IEEE Transactions on Pattern Analysis and Machine Intelligence, Vol. 15, No. 11, 1993, pp. 1131-1147. http://dx.doi.org/10.1109/34.244675

[3] H. K. Park and M. J. Chung, "External Force of Snake: Virtual Electric Field,” Electronics Letters, Vol. 38, No, 24, 2002, pp. 1500-1502.

http://dx.doi.org/10.1049/el:20021037 
[4] X. Xie and M. Mirmehdi, "Magnetostatic Field for the Active Contour Model: A Study in Convergence," Proceedings of the 17th British Machine Vision Conference, Edinburgh, 4-7 September 2006, pp. 127-136.

[5] K. W. Sum and Paul Y. S. Cheung, "Boundary Vector Field for Parametric Active Contours," Pattern Recognition, Vol. 40, No. 6, 2007, pp. 1635-1645. http://dx.doi.org/10.1016/j.patcog.2006.11.006

[6] B. Li and S. T. Acton, "Active Contour External Force Using Vector Field Convolution for Image Segmentation," IEEE Transactions on Image Processing, Vol. 16, No. 8, 2007, pp. 2096-2106. http://dx.doi.org/10.1109/TIP.2007.899601

[7] C. $\mathrm{Xu}$ and J. L. Prince, "Generalized Gradient Vector Flow External Forces for Active Contours,” Signal Processing, Vol. 71, No. 2, 1998, pp. 131-139. http://dx.doi.org/10.1016/S0165-1684(98)00140-6

[8] C. Xu and J. L. Prince, "Snakes, Shapes, and Gradient Vector Flow,” IEEE Transactions on Image Processing, Vol. 7, No. 3, 1998, pp. 359-369. http://dx.doi.org/10.1109/83.661186

[9] X. Xie and M. Mirmehdi, "RAGS: Region-Aided Geometric Snake,” IEEE Transactions on Image Processing, Vol. 13, No. 5, 2004, pp. 640-652. http://dx.doi.org/10.1109/TIP.2004.826124

[10] N. Ray and S. T. Acton, "Motion Gradient Vector Flow:
An External Force for Tracking Rolling Leukocytes with Shape and Size Constrained Active Contours," IEEE Transactions on Medical Imaging, Vol. 23, No. 12, 2004, pp. 1466-1478. http://dx.doi.org/10.1109/TMI.2004.835603

[11] H. Park, T. Schoepflin and Y. Kim, “Active Contour Model with Gradient Directional Information: Directional Snake," IEEE Transactions on Circuits and Systems for Video Technology, Vol. 11, No. 2, 2001, pp. 252-256. http://dx.doi.org/10.1109/76.905991

[12] G. Zhu, S. Zhang, Q. Zeng and C. Wang, “Gradient Vector Flow Active Contours with Prior Directional Information,” Pattern Recognition Letters, Vol. 31, No. 9, 2010, pp. 845-856. http://dx.doi.org/10.1016/j.patrec.2010.01.011

[13] G. Zhu, S. Zhang, X. Chen and C. Wang, "Novel Gradient Vector Flow-Based Balloon Force for Active Contours," Journal of Electronic Imaging, Vol. 18, No. 2, 2009, pp. 023007-1-023007-8. http://dx.doi.org/10.1117/1.3132005

[14] M. S. Hassouna and A. A. Farag, "Variational Curve Skeletons Using Gradient Vector Flow,” IEEE Transactions on Pattern Analysis and Machine Intelligence, Vol. 31, No. 12, 2009, pp. 2257-2274. http://dx.doi.org/10.1109/TPAMI.2008.271 